\title{
Applications of the microprocessor in the behavioral medicine laboratory
}

\author{
ROBERT S. DAVIDSON and ROBERT F. BREMSER \\ Veterans Administration Medical Center, Miami, Florida 33125
}

\begin{abstract}
Applications of the microprocessor for use within a behavioral medicine laboratory are reviewed. Current uses include clinical assessment (test administration, scoring, and intepretation), patient data file creation, data storage and retrieval, word processing of manuscripts and reference lists, data reduction, statistical analysis, and computer-assisted therapy. Instrumentation and software in use and under development are described, as well as future prospects.
\end{abstract}

Computers are being used increasingly in mental health facilities (Johnson \& Williams, 1975). Several systems of medical and psychiatric interviewing have been developed, often with the goal of providing diagnostic information (Angle, Hay, Hay, \& Ellinwood, 1977; Kleinmuntz, 1972; Mayne, Weksel, \& Sholtz, 1968; Reich, Robins, Woodruff, Taibleson, Rich, \& Cunningham, 1975; Schoolman \& Bernstein, 1978; C. W. Slack, 1968). There has also been an increasing use of computer systems in experimental psychology laboratories, at first mainly for statistical analysis, and then later for the on-line, experimental control of programs (Martin \& Conner, 1975; Schneider \& Scholz, 1973; Shure \& Meeker, 1970; Starr \& Flanik, 1977). Most of the early work made use of full-sized computers that were too costly for many facilities. Within the last several years, the development of the microprocessor has made computer hardware and software more costeffective and, therefore, more accessible to a growing number of facilities. This article reviews a number of functions within a behavioral medicine laboratory that have been computer assisted, as well as many more that are planned or in progress.

\section{INSTRUMENTATION}

Instrumentation for this program has been described by Bremser and Davidson (1978). The microprocessor of primary use is an 8080 system (Sol Terminal Computer, Processor Technology) with 48K RAM and onboard circuitry that includes parallel and serial input/ output (I/O) ports with full-control handshaking and a switch-selectable baud rate of 75-9,600, direct connections for a video monitor, and a built-in keyboard with a 128 upper- and lowercase alphanumeric character generater with the ASCII control character set. Periph-

The program reported here has been supported by VA Research Project 1777-01, "Behavioral Medicine: Assessment and Treatment of Alcoholism," Robert S. Davidson, prinicpal investigator. erals include a video monitor, two disk-drive units and floppy-disk control boards (North Star), a printer (OKIdata 110), and an IBM Selectric typewriter. The typewriter has been modified to include solenoids and a special control interface board of our design that drives the appropriate solenoid configuration for a specific character output from the computer through a standard parallel output port. The disk control units allow access to $90 \mathrm{~K}$ additional memory per disk. Like many other systems of this size and capability, this computer uses a disk operating system (North Star DOS) that, when combined with one of the recently extended versions of BASIC, enables some very powerful operations.

A second microprocessor (Byte 8) that has $40 \mathrm{~K}$ of onboard memory, an A/D converter, one disk drive, and three parallel and one serial $\mathrm{I} / \mathrm{O}$ ports is also available. This smaller computer is used primarily for creating new programs, accessing subject files, and performing some statistical analyses.

\section{SOFTWARE}

All of our programs are written in extended BASIC. BASIC was chosen because it is easy to program, it was designed for human interactional programming, it enables manipulation of strings of alphanumeric characters, and it is interactive.

\section{CLINICAL ASSESSMENT}

The basic orientation of behavioral medicine is a multidisciplinary and holistic approach to the multitude of factors involved in the etiology, treatment, and prevention of chronic ailments, such as cardiovascular diseases, cancer, psychosomatic disorders, and alcoholism. Because of this orientation, it is usually important to collect information about many areas of an individual's life in order to determine his status with regard to risk factors for disease, contributory factors, overall health, physical fitness, strengths, and motivation for treatment. Frequently, assessment is directed toward such 
factors in medical, behavioral, physiological, cognitive, and motivational areas. An appropriate summary of such data often provides a well-rounded description that immediately suggests treatments of choice. The computer is ideally suited to assist in this massive undertaking.

The computer can save personnel time by administering assessment devices. Routines for the administration of typical psychological tests are easily programmed. We have automated the administration of the Millon Clinical Multiaxial Inventory (MCMI), Alcoholic Evaluation Instrument (Alc Eval), Self-Control Inventory, Cornell Medical Index, Multiple Affect Adjective: Check List, Profile of Mood States, and Locus of Control Scale.

Programming administration of the tests was simple. Many of them consist entirely of true-false or yes-no items, the scores of which contribute to a total score or to several individual scales. In each case, the program presents one item at a time to the examinee, who is requested to indicate his/her response by pressing a letter on the keyboard (e.g., " $T$ " for true, "F" for false). Objective, multiple-choice items are similar, but they are answered by selecting a number (e.g., 1-4).

A small machine language routine callable from BASIC has been developed that has proved very effective in helping to eliminate user error. This routine flashes the word "ERROR" in capital letters the size of the full screen following any input other than an instructed one. The user is then informed that an input error has been made and must be corrected before more data can be entered.

Test interpretation is much more difficult; automated interpretation programs are available only for the MMPI and several other tests. On simple tests with individual scales that can be interpreted independently, designing a computer program for the interpretation consists of matching phrases or statements to individual scale scores. With more complex tests, such as the MMPI, the problem is compounded by the necessity of pattern or profile analysis. In other words, scores on individual scales are not independent and are interpreted differently, depending on scores on other scales. Therefore, our efforts in this area have been limited.

Broad-ranging assessment within behavioral, physiological, cognitive, attitudinal, and motivational areas is helpful to (1) find factors responsible for the problem, (2) determine whether treatment has effected change in all important areas, and (3) better match particular treatments to individuals (Ciminero, Calhoun, \& Adams, 1977; Cone \& Hawkins, 1976; Hersen \& Bellack, 1976). Many broad-ranging, holistic assessment programs now use test batteries that combine a personality test, a symptom checklist, a mood scale, and measures that are tailored specifically for assessment of a particular target of behavior.

Our programs create a separate file for all of the tests used in a battery, so that each can be separately accessed. Such files facilitate the study of tests of differences between means (e.g., $t$ tests), correlations, and regression. Programs have been written to conduct such tests automatically, without need for further data reduction or manipulation.

One program has been written that provides zeroorder correlations on all possible pairs of variables in the patient data file pool. This program is useful in determining the reliability of items within the assessment battery. For example, it will retrieve the data from two test administrations, calculate the correlations, and print them out. In execution, this program requests the type of statistic to be calculated and the names of the variables and then uses main identification files to find the location of each variable. It informs the user of this location and makes a temporary file of the data to be used for data manipulation and analysis. This methodology protects the main data file and facilitates access from locations on different disks.

In addition, many complex statistical manipulations beyond the capability of the microprocessor are available using remote communication to a large computerbased statistical package (Dixon, 1973; Nie, Hull, Jenkins, Steinbrenner, \& Bent, 1975). For example, in predicting treatment attrition and outcome from the assessment variables discussed previously, we frequently use statistics such as multiple regression and discriminant function which are more cost effective on a large computer system.

\section{WORD PROCESSING}

Word processing capability makes available a powerful set of operations that are useful in the preparation of manuscripts, in storing hard copies of patient data and psychological reports, and in keeping up-to-date reference lists. Word processing allows the manipulation of inputs that are sent from the keyboard in a mainer analogous to standard typing. However, the text is displayed on the CRT prior to printout. With additional keyboard command functions, it is possible to move the cursor to any point in the displayed text and to insert or delete characters very rapidly. These functions make editing the displayed text quick and easy. Another valuable function for the word processor is maintenance of a list of references.

\section{COMPUTER-ASSISTED THERAPY}

Several attempts to computerize psychotherapy have been reported, but they have not been considered successful (Colby, 1980; Meany, 1962; C. W. Slack, 1960; Weizenbaum, 1966). At least some of the lack of success has been due to the fact that the programs thus far have been very elementary, lacking in systematic conceptual bases and limited to interacting with patients 
in standard and very limited modes of response. For example, LIZA (Weizenbaum, 1966) was an attempt to simulate Rogerian nondirective counseling, especially by being nonjudgmental, by reflecting the feelings of the client, and by providing empathy and genuineness in a nondirective manner. Inputs that did not fall within the province of the program (which were many) received the computer output "Please go on." Similarly, Colby, Watt, and Gilbert (1966) met with resistance to a program containing a limited number of possible responses to 500 acceptable keyed inputs. This model also sought to evoke and reflect feelings and to interpret statements of the interviewee. Both the Colby and Weizenbaum systems attempted to simulate natural language interactions as might occur in "dynamic" forms of therapy. The major problem with these research efforts was the attempt to simulate a natural language with a conversational and yet interpretive exchange.

Several less comprehensive computer-assisted programs of education or therapy have had better success. For example, computer-controlled biofeedback conditioning of human heart rate has proved useful (Lang, 1969, 1974).

We have recently been working on computer-assisted programs of education and prevention in alcoholism. Some educational researchers (Cameron, Campbell, \& Cipywnyk, 1977; Cappiello, 1977) have suggested the possibility of secondary prevention by teaching patients about etiology, symptomatology, and therapy. patients about etiology, symptomatology, and therapy. Although these programs proposed guidelines, neither dealt directly with patients.

We are using a programmed learning format to design an interactive system to teach patients about the nature and extent of their disorders, risk factors and causes, symptoms and effects, types of treatment available, and certain specific behavior control approaches. We pretest each patient on each unit to be learned and bypass some or all of the material contingent on early mastery. We also match or emphasize portions of the recommended therapy units, depending on early or prior responses. For example, with an alcoholic patient whose history has already demonstrated periods of control alternating with periods of extended intoxication, it is possible to emphasize portions of the program designed to instruct and teach the habits of maintained controlled (subintoxicating) drinking.

We anticipate that attitudinal, motivational, and physiological responses will be added to our therapy program. This will broaden the base of the therapy program in the same way that our assessment work will. For example, it might be helpful to provide relaxation training for those patients who are high in measured muscle tension. The computer could provide measures of muscle tension during relaxation training, as in biofeedback. Similarly, attitudinal and cognitive manipulations might assist patients in eliminating irrational beliefs and thoughts that have led to drinking in the past.

\section{DISCUSSION}

Computer systems comparable to ours are more than adequate for assessment, word processing, patient tracking and record keeping, data analysis, and many other related applications. However, we have found that the purchase and installation of a computer system has led to demands for increased capabilities. For example, it would be desirable in our laboratory to be able to provide computer-assisted assessment for more than one patient at a time, and perhaps at the same time to make available access to the computer for programming and statistical analysis. With the systems described, only one of these functions is available on each of the two systems at any one time.

Recently developed microprocessors with multiuser capabilities have become available. Several manufacturers (Alpha Micro, Cromemco, Ohio Scientific, etc.) have designed systems that can simultaneously perform most of the functions described above. These systems perform at speeds comparable to minicomputer systems and range in price upward from $\$ 15,000$, depending on peripherals. For example, an Alpha Micro equipped with a 10-megabyte hard disk system, six terminals, and a good quality high-speed printer costs approximately $\$ 30,000$. An equivalent minicomputer system, the DEC $11 / 34$, similarly configured, would cost about $\$ 55,000$. All of these systems have the capability to handle inputs from six terminals simultaneously. Thus, for example, three patients could be being assessed, one person could be programming, one person could be word processing, and one person could be doing statistical analyses.

\section{REFERENCES}

Angle, H. V., Hay, L. R., Hay, W. M., \& Ellinwood, E. H. Computer assisted behavioral assessment. In J. D. Cone \& R. P. Hawkins (Eds.), Behavioral assessment. New York: Brunner/Maazel, 1977.

Bremser, R. F., \& Davidson, R. S. Microprocessor-assisted assessment in the clinical research laboratory. Behavior Research Methods \& Instrumentation, 1978, 10, 582-584.

Cameron, O. N., Campbell, A. R., \& Cipywnyk, D. Alcoholism: A new approach to an old problem. Canadian Journal of Public Health, 1977, 68, 483-485.

Cappiello, L. A. Prevention of alcoholism: A teaching strategy. Journal of Drug Education, 1977, 7, 311-316.

Ciminero, A. R., Calhoun, K. S., \& Adams, H. E. (Eds.). Handbook of behavioral assessment. Somerset, N.J: Wiley Interscience, 1977.

Colby, K. M. Computer psychotherapists. In J. B. Sidowski, J. H. Johnson, \& T. A. Williams (Eds.), Technology in mental health care delivery systems. Norwood, N.J: Ablex, 1980.

Colby, K. M., WATt, J. B., \& Gilbert, J. P. A computer method of psychotherapy: Preliminary consideration. Journal of Nervous and Mental Disease, 1966, 142, 148-152.

Cone, J. E., \& Hawkins, R. P. (Eds.), Behavioral assessment. New York: Brunner/Maazel, 1977. 
Dixon, W. J. (Ed.). BMD: Biomedical computer programs. Berkeley: University of California Press, 1973.

Hersen, M., \& Bellack, A. (Eds.). Behavioral assessment: A practical handbook. New York: Pergamon Press, 1976.

Johnson, J. H., \& Williams, T. The use of on-line computer technology in a mental health admitting system. American Psychologist, 1975, 30, 388-390.

KLeinmuntz, B. Computers in personality assessment. New York: General Learning Press, 1972.

LANG, P. J. The on-line computer in behavior therapy research. American Psychologist, 1969, 24, 236-239.

LANG, P. J. Learned control of human heart rate in a computer directed environment. In P. A. Obrist, A. H. Black, J. Brener, \& L. V. DiCara (Eds.), Cardiovascular psychophysiology. Chicago: Aldine, 1974.

Martin, R. A., \& Conner, R. J. Computer control for operant oriented laboratories. Behavior Research Methods \& Instrumentation, 1975, 7, 151-156.

Mayne, J. G., Weksel, W., \& Sholtz, P. N. Toward automating the medical history. Mayo Clinic Proceedings, 1968, 43, 1-25.

Nie, N. H., Hull, C. H., Jenkins, J. G., Steinbrenner, K., \& BENT, D. H. Statistical package for the social sciences (2nd ed.).
New York: McGraw-Hill, 1975.

Reich, T., Robins, K. N., Woodruff, R. A. Taibleson, M., Rich, C., \& Cunningham, L. Computer-assisted derivation of a screening interview for alcoholism. Archives of General Psychiatry, 1975, 32, 847-852.

Schnetde R, W., \& Scholz, K. W. Requirements for minicomputer operating systems for human experimentation and an implementation of a 4K PDP-8 computer. Behavior Research Methods \& Instrumentation, 1973, 5, 173-177.

Schoolman, H. M., \& Bernstein, L. M. Computer use in diagnosis, prognosis, and therapy. Science, 1978, 200, 926-931.

Shure, G. H., \& MEeken, R. J. A computer-based experimental laboratory. American Psychologist, 1970, 25, 962-969.

Slack, C. W. Experimenter-subject psychotherapy: A new method of introducing intensive office treatment for unreachable cases. Mental Hygiene, 1960, 44, 238-256.

Stark, B. J., \& Flanik, S. A. ALL: Automated learning laboratory. Behavior Research Methods \& Instrumentation, 1977, 9, 189-192.

Weizenbaum, J. A computer program for the study of natural language communication between man and machine. Communications of the $A C M, 1966,9,36-45$. 